\title{
Educational Software Audit
}

\section{Auditoría de software educativo}

\author{
Anis F. Galimyanov, Alina I. Muzafarova \\ ${ }^{1}$ Kazan Federal University \\ e-mail:anis_59@mail.ru,Alina291997muzafarova@yandex.ru \\ Tel.: +79050210915
}

Enviado: 27 de junio de 2019

Aceptado para publicar: 30 de julio de 2019

Publicado: 8 de agosto de 2019

\begin{abstract}
This article describes an audit in the IT field, namely the audit of software using the example of Kazan (Volga Region) Federal University. The most significant goal of the audit is to discover the full list of programs installed on the enterprise's machines. This list should include all software products without exception: both used by employees to solve their daily questions, and those programs that were not used at all, but simply downloaded for interest. It is noted, including incorrectly deleted software that has retained its fingerprints in the system. In the work, a comparison was made of the most well-known and used products of Microsoft System Center Configuration Manager and Kaspersky Security Center and a comparative integration of all the information that collects these funds was performed. Collecting and processing data on the software installed on the machines of an educational institution is a time-consuming task. The system of automated software audit connects remotely to computers on the local network and accumulates data about the software installed on them. To improve this process, a program with the necessary interface has been written, which allows obtaining a database that contains data about all computers with the software installed on them. This will further make it possible to formulate recommendations for the further development of the computer park on the example of KPU.
\end{abstract}

Keywords: audit, inventory, software, Microsoft System Center Configuration Manager, Kaspersky Security Center, control, software, database, DBMS

Este artículo describe una auditoría en el campo de TI, es decir, la auditoría de software utilizando el ejemplo de la Universidad Federal de Kazan (Región del Volga). El objetivo más importante de la auditoría es descubrir la lista completa de programas instalados en las máquinas de la empresa. Esta lista debe incluir todos los productos de software sin excepción: ambos utilizados por los empleados para resolver sus preguntas diarias, y aquellos programas que no se utilizaron en absoluto, sino que simplemente se descargaron por interés. Cabe señalar, incluido el software eliminado incorrectamente que ha conservado sus huellas digitales en el sistema. En el trabajo, se realizó una comparación de los productos más conocidos y usados de Microsoft System Center Configuration Manager y Kaspersky Security Center y se realizó una integración comparativa de toda la información que recolecta estos fondos. Recopilar y procesar datos en el software instalado en las máquinas de una institución educativa es una tarea que requiere mucho tiempo. El sistema de auditoría de software automatizado se conecta de forma remota a las computadoras en la red local y acumula datos sobre el software instalado en ellas. Para mejorar este proceso, se ha escrito un programa con la interfaz necesaria, que permite obtener una base de datos que contiene datos sobre todas las computadoras con el software instalado en ellas. Esto permitirá formular recomendaciones para un mayor desarrollo del parque de computadoras en el ejemplo de KPU.

Palabras clave: auditoría, inventario, software, Administrador de configuración de Microsoft System Center, Kaspersky Security Center, control, software, base de datos, DBMS. 


\section{Introduction}

As noted in [1], one of the important components of market relations is control. Control can be both state and independent. Independent control is called an audit. The concept of "audit" comes from the Latin word audio - I listen. The main objective of the audit is to ensure the control of the accuracy of the information.

Software audit is the study of all software installed on the organization's network equipment. The audit makes it clear how software is used effectively, and what opportunities there are to improve this performance. The software audit is carried out in four necessary steps:

1. Setting tasks

2. Collect all required information and data

3. Analysis of acquired information

4. Registration of the results in the form of a report

All these tasks can be carried out both manually and automatically using special software.

Audit of the organization's software is designed to solve the following tasks:

- establishing the legality of the software used on the company's computers to prevent penalties;

- determination of the compliance of established programs with business processes and goals of the company and increasing their efficiency;

- reducing or optimizing software maintenance costs; increasing the level of security of the company's information networks.

The result of the audit is the creation of a list of documentation containing detailed information about the software installed on the organization's computers, as well as a set of proposals for improving the quality of operations, improving security, security and efficient use of the software. This paper presents an IT software audit using the example of Kazan (Volga Region) Federal University and outlines methods for constructing services for effective software auditing.

\section{Methods and Tools}

One of the important tasks of the staff of the System Administration Department of the Telecommunications and Information Systems Engineering Center is the collection of data on the software installed on electronic devices of the Kazan Federal University.

Currently, ISTIS KFU employees collect information using products such as Microsoft System Center and Kaspersky Security Center. The collection and processing of data on the software installed on the machines of Kazan Federal University is a time-consuming task. The system of automated audit software KFU remotely connected to computers on the local network and accumulates data about the software installed on them. Schemes of the system of automated audit software KFU presented in Figure 1.

Designation: User - user, Collection of information - Information collection, View information - Software selection - Software selection, getting a schedule - Receiving a schedule, receiving reports - Receiving reports.
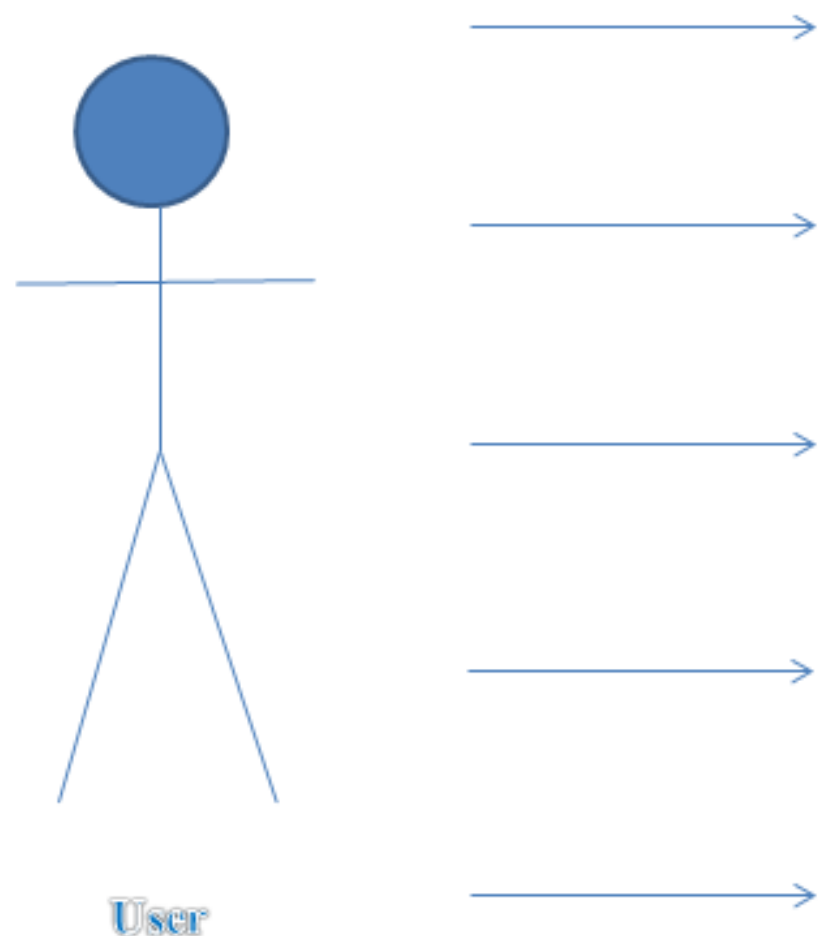

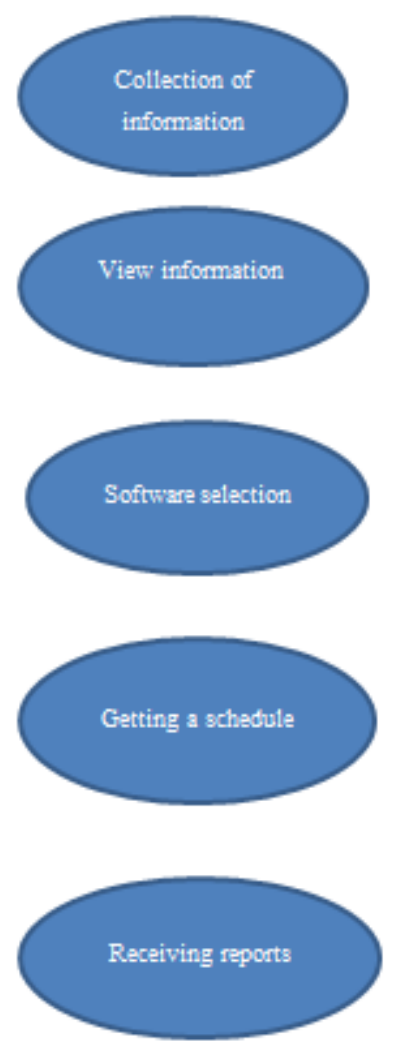


Fig.1 The system of automated audit software KFU The main problem encountered by the employees of the system administration department when conducting an audit of software is the lack of integrity and completeness of data. This paper compares the two main audit products and integrates all the information that these funds collect. Consider in brief these products.

\section{Microsoft System Center Configuration Manager}

Microsoft's System Center Configuration Manager is a tool for centrally updating and deploying applications, auditing computers and software, and monitoring. [2,17] The product also includes Asset Intelligence. Asset Intelligence compiles installed software with support for software agents. The automatically updated catalog contains a description of more than 300 thousand items from different manufacturers.

SCCM includes workstation management tools. This software product is used in a complex IT infrastructure.

System Center Configuration Manager organizes software distribution from a single console, counts programs, compares installed and authentic configuration values, scales the conclusion, minimizes risks, makes it possible to distinguish security problems, remotely connects to a computer from a common console, updates offline, interacts with System Center Virtual Machine Manager and provides a service of virtual machines stored in the library.

This product is licensed by KPU since the automatically updated catalog contains a description of more than 300 thousand items from different manufacturers. Catalog and categories can be updated independently. AI has special import tools, server licensing resources, processor licenses, etc. The price of the System Center Configuration Manager, which includes the AI, is approximately $\$ 40$ for the license you need to buy for each computer. Of course, only the Windows platform is supported.

\section{Kaspersky Security Center}

Kaspersky Security Center is a mechanism for managing a security system that gives a system administrator access to detailed information about the degree of network security. [3] This powerful and convenient software product helps to ensure the correct operation of the company's IT infrastructure and increase the productivity of its business.

In addition to detailed control over the security of the IT infrastructure, KSC contains system administration tools that provide an opportunity to increase efficiency and reduce operating costs.

Kaspersky Security Center allows you to adjust the operating system images: create, copy and deploy.

In Kaspersky Security Center there is a function of remote installation of software, which saves time for system administrators and reduces the size of the traffic transmitted over the network.

Kaspersky Security Center allows you to manage software and monitor software licenses. This software product contains the function of automatic accounting of hardware. To monitor the programs used in the KSC there is the possibility of creating reports. [4,16]

Consider the use of the described tools for auditing.

To use System Center Configuration Manager, you need to connect to a virtual private network (VPN). By completing the VPN connection to the kpfu corporate network, you can use SCCM. With the help of the System Center Configuration Manager software product, it is possible to view information about computer hardware and software of Kazan Federal University installed on them. During the work, it was found that more than 5,000 computers are used at the KFU.

SCCM software product allows you to view information about used computers. In particular, using this program, you can get the computer name, information about the hardware, information about the software, etc. Figure 2 shows one of the SCCM screens. 


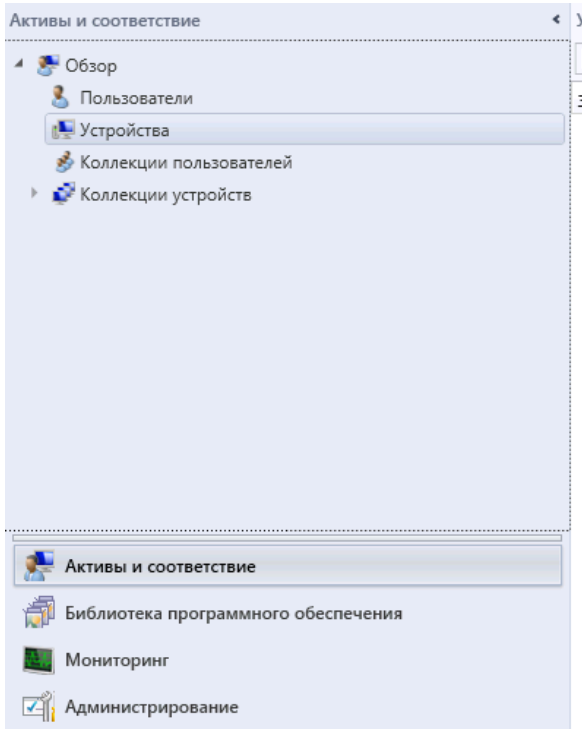

\begin{tabular}{|c|c|c|c|c|}
\hline \multicolumn{5}{|l|}{ Поиск } \\
\hline Значок & 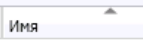 & Клиент & Код сайта & Активность клиентов \\
\hline $\mathbf{E}$ & CLASS-05-102-14 & Нет & PRM & \\
\hline 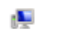 & CLASS-12-305-01 & Да & PRM & Активное \\
\hline $\mathbf{E}$ & CLASS-12-307-01 & Нет & PRM & \\
\hline $\mathbf{E}$ & CLASS-13-211-01 & да & PRM & Активное \\
\hline $\mathbf{t}$ & CLASS-14-108-01 & дa & PRM & Активное \\
\hline $\mathbf{E}$ & CLASS-14-109-01 & да & PRM & Активное \\
\hline $\mathbf{E}$ & CLASS-14-1213-1 & $\mathrm{Aa}$ & PRM & Активное \\
\hline $\mathbf{5}$ & CLASS-14-1311-0 & да & PRM & Активное \\
\hline $\mathbf{E}$ & CLASS-14-1313-1 & да & PRM & Неактивное \\
\hline $\mathbf{E}$ & CLASS-14-216-01 & да & PRM & Активное \\
\hline $\mathbf{2}$ & CLASS-14-218-02 & да & PRM & Активное \\
\hline $\mathbf{E}$ & CLASS-14-309-01 & да & PRM & Активное \\
\hline $\mathbf{F}$ & CLASS-14-312-01 & $\mathrm{Aa}$ & PRM & Активное \\
\hline $\mathbf{F}$ & CLASS-14-402-01 & да & PRM & Активное \\
\hline $\mathbf{E}$ & CLASS-14-403-01 & $\mathrm{Aa}$ & PRM & Активное \\
\hline $\mathbf{E}$ & CLASS-14-404-01 & да & PRM & Активное \\
\hline $\mathbf{E}$ & CLASS-14-409-01 & да & PRM & Активное \\
\hline $\mathbf{E}$ & CLASS-14-411-02 & да & PRM & Неактивное \\
\hline $\mathbf{E}$ & CLASS-16-310-01 & Нет & PRM & \\
\hline
\end{tabular}

Fig. 2. Issuance of information about customer activity.

With the help of the SCCM program, it is possible to make queries in the SQL language and build various reports on these requests. Figure 3 presents a request for a sample of all software, versions and software manufacturers.

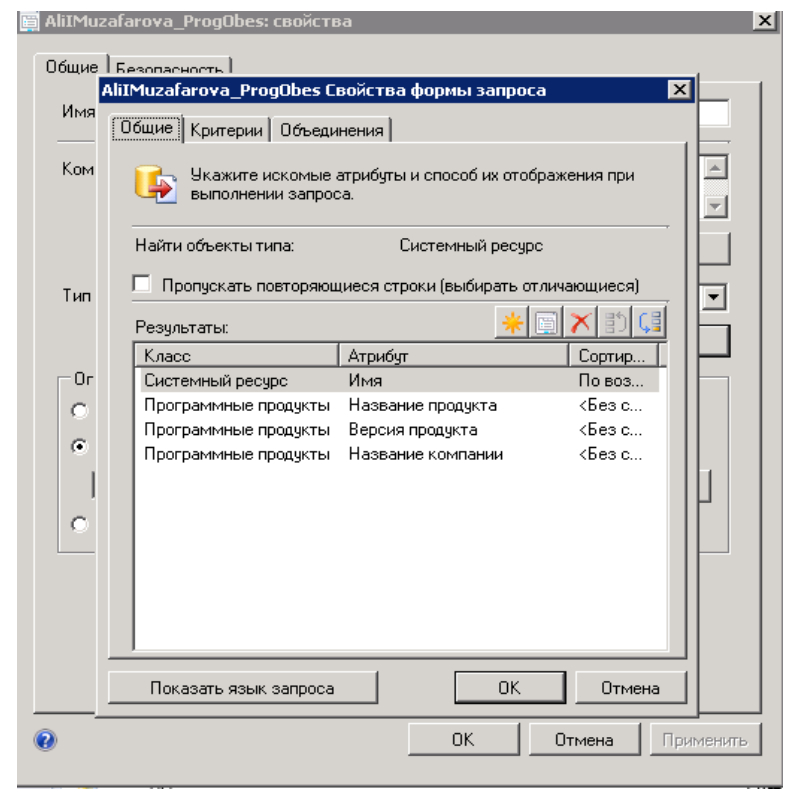

Fig. 3. Request for selection of all software, versions and software manufacturers

The following query was created for selecting software, versions and manufacturers in MS SQL: Select

CM PRM.dbo.[vSMS R System].[Name0],CM PRM.dbo.vSMS_G_System_SoftwareProduct. [ProductName],CM_PRM.dbo.vSMS_G_System SoftwareProduct.[ProductVersion],CM_PRM.dbo .vSMS_G_System_SoftwareProduct.CompanyNa me

FROM

CM_PRM.dbo.vSMS_R_System,CM_PRM.dbo.v SMS G System SoftwareProduct Where CM_PRM.dbo.vSMS_R_System.ItemKey $=$

CM PRM.dbo.vSMS G System SoftwareProduc t.ClientId

Order by

CM_PRM.dbo.[vSMS_R_System].[Name0]

The query results were exported to excel (Fig. 4). It turned out 533178 different software products. 


\begin{tabular}{|c|c|c|c|c|}
\hline$\Delta$ & A & B & $c$ & D \\
\hline 108607 & DVS-05-217-05 & SetACL & $2,1,3,0$ & Helge Klein \\
\hline 108608 & DVS-05-217-05 & Microsoft Windows Operating System & 5.5 .0031 .0 & Microsoft Corporation \\
\hline 108609 & DVS-05-217-05 & Microsoft Setup Bootstrapper & 14.0.7011.1000 & Microsoft Corporation \\
\hline 108610 & DVS-05-217-05 & Microsoft Office 2010 & 14.0.7006.1000 & Microsoft Corporation \\
\hline 108611 & DVS-05-217-05 & Microsoft Office 2010 & 14.0.6009.1000 & Microsoft Corporation \\
\hline 108612 & DVS-05-217-05 & Watson Subscriber for SENS Network Notification & 14.0.7011.1000 & Microsoft Corporation \\
\hline 108613 & DVS-05-217-05 & Microsoft $^{\ominus}$ Visual Studio $^{\odot} 2010$ & 10.0 .40305 .0 & Microsoft Corporation \\
\hline 108614 & DVS-05-217-05 & Microsoft Office 2010 & 14.0.7011.1000 & Microsoft Corporation \\
\hline 108615 & DVS-05-217-05 & Microsoft Office 2010 & 14.0.6022.1000 & Microsoft Corporation \\
\hline 108616 & DVS-05-217-05 & DSConfig & 1.0 .0 .1 & MONOGRAM Multimedia, s.r.o. \\
\hline 108617 & DVS-05-217-05 & Microsoft Office 2010 & 14.0.7010.1000 & Microsoft Corporation \\
\hline 108618 & DVS-05-217-05 & Microsoft OneNote & 14.0.6009.1000 & Microsoft Corporation \\
\hline 108619 & DVS-05-217-05 & Adobe Acrobat text extractor for non-PDF files & 11.0.0.379 & Adobe Systems Incorporated \\
\hline 108620 & DVS-05-217-05 & Microsoft Office 2010 & 14.0 .7007 .1000 & Microsoft Corporation \\
\hline 108621 & DVS-05-217-05 & Microsoft Office Help Viewer & 14.0.7010.1000 & Microsoft Corporation \\
\hline 108622 & DVS-05-217-05 & Microsoft Application Error Reporting & 14.0.7011.1000 & Microsoft Corporation \\
\hline 108623 & DVS-05-217-05 & Microsoft Office 2010 & 14.0.7005.1000 & Microsoft Corporation \\
\hline 108624 & DVS-05-217-05 & Microsoft Office 2010 & 14.0.7005.1000 & Microsoft Corporation \\
\hline 108625 & DVS-05-217-05 & Microsoft Office 2010 & 14.0.6108.5000 & Microsoft Corporation \\
\hline 108626 & DVS-05-217-05 & SelfCert & 14.0.7011.1000 & Microsoft Corporation \\
\hline 108627 & DVS-05-217-05 & Bootstrapper Small & 11.0 .0 .379 & Adobe Systems Incorporated \\
\hline 108628 & DVS-05-217-05 & Intel(R) Graphics & $1,5,47,0$ & Intel Corporation \\
\hline 108629 & DVS-05-217-05 & Intel(R) Installation Framework & $1,4,1,0$ & Intel Corporation \\
\hline
\end{tabular}

Fig.4. Query results in excel from SCCM

Similar actions were performed in Kaspersky Security Center. In the MS SQL DBMS, a request was made to select all software, versions and software producers installed on computers of the Kazan Federal University.

select

h.wstrDisplayName,

a.[wstrPublisher], a.wstrDisplayName,
a.wstrBuild

from

KAV.dbo.v_akpub_host as $\mathrm{h}$

INNER JOIN

KAV.dbo.v akpub application as a

ON h.nId = a.nHostId

ORDER BY h.wstrDisplayName ASC

Results of export to excel, 324621 different software products:

\begin{tabular}{|c|c|c|c|}
\hline MICROSOF-408452 & J-Link ARM V4.59d & V4.59d & SEGGER Microcontroller Systeme GmbH \\
\hline КА-ПК & ScanSnap Organizer & V5.1L42 & PFU \\
\hline ИРИНА-ПК & SAMSUNG Android USB Modem Software & V5.28.2.1 & \\
\hline КА-ПК & CardMinder & V5.2L20 & PFU \\
\hline NEWWORKER & Drive Space Indicator v5.3.5.5 & v5.3.5.5 & DougieFresh's \\
\hline IF-12-1109-15 & Drive Space Indicator 5.3.6.4 & v5.3.6.4 & DouglasSoft LTD \\
\hline IGNGT-13-111-06 & Auslogics BoostSpeed & v5.4.0.10 & alert30 \\
\hline DO-01-114-01 & ScanSnap Manager & V5.5L10 & PFU \\
\hline UMU-02-110B-01 & ScanSnap Manager & V5.5L10 & PFU \\
\hline UMU-01-114-03 & ScanSnap Manager & V5.5L10 & PFU \\
\hline DO-01-116-18 & ScanSnap Manager & V5.5L10 & PFU \\
\hline IFMB-04-38-02 & MiraScan 6.1(5550 series) & V6.1(5550 series) & Benq Scan \\
\hline КА-ПК & ScanSnap Manager & V6.2L24 & PFU \\
\hline DO-01-123-18 & ScanSnap Manager & V6.5L40 & PFU \\
\hline IFMB-11-203-01 & mp3Tag Pro & v7.2.0.526 & ManiacTools \\
\hline IFI-33-209B-03 & Total Commander & v7.56 Final & oszone.net \\
\hline IFI-33-122-02 & Total Commander & v7.56 Final & oszone.net \\
\hline IF-12-101B-06 & ALZip 8.51 & v8.51 & ESTsoft Corp. \\
\hline OFFICE-1 & VueScan 9.0.57 & v9.0.57 & (C) ZverDvD \\
\hline IFMB-11-203-01 & VueScan 9.0.61 & v9.0.61 & Hamrick Software \\
\hline MYCOMP & UltralSO & v9.3.5 Build 2716 & oszone.net \\
\hline IVMT-14-1206-01 & TeXnicCenter Version 1.0 Stable RC1 & Version 1.0 Stable RC1 & TeXnicCenter.org \\
\hline IVMT-14-1206-08 & TeXnicCenter Version 1.0 Stable RC1 & Version 1.0 Stable RC1 & TeXnicCenter.org \\
\hline
\end{tabular}

Fig. 5. Query results in excel from KSC

Next, data was imported from the received data collection sources into a new database using the SQL Server Import and Export Wizard. (pic.6) 


\begin{tabular}{|c|c|c|c|c|c|c|}
\hline \multicolumn{4}{|c|}{ Мастер импорта и зкспорта SQL Sevver $\quad$ - } & \multicolumn{3}{|c|}{$\begin{array}{l}\text { Fig. 6. Importing Data Using SQL Server Import } \\
\text { and Export Wizard }\end{array}$} \\
\hline \multicolumn{4}{|c|}{ Выполнение прошло успешно } & & & \\
\hline \multicolumn{2}{|c|}{ ( } & $\begin{array}{l}11 \text { Всего } \\
11 \text { Успешно }\end{array}$ & $\begin{array}{l}\text { О Ошибка } \\
0 \text { Внимание }\end{array}$ & \multirow{10}{*}{\multicolumn{3}{|c|}{$\begin{array}{l}\text { As a result of the import, two tables wer } \\
\text { obtained: dbo. Kaspersky, dbo.SCCM } \\
\text { Further, with the help of the following query, tw } \\
\text { tables were combined into one, which was calle } \\
\text { dbo.KS (Fig. 7) } \\
\text { Request: } \\
\text { SELECT * INTO[KS]FROM [Diploma (Finishe } \\
\text { plates)].[dbo].[SCCM] } \\
\text { UNION SELECT *FROM [Diploma (Read } \\
\text { plates)].[dbo].[ Kaspersky] }\end{array}$}} \\
\hline \multicolumn{4}{|c|}{ Подробности: } & & & \\
\hline \multirow{2}{*}{\multicolumn{2}{|c|}{$\begin{array}{l}\text { Действие } \\
\text { Иницализация задачи потока данныьх }\end{array}$}} & 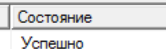 & Coobuethe & & & \\
\hline & & $\begin{array}{l}\text { Успешно } \\
\text { Успешно }\end{array}$ & & & & \\
\hline 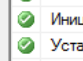 & ка команд SQL & Успешно & & & & \\
\hline \multirow{5}{*}{\multicolumn{2}{|c|}{$\begin{array}{l}\text { Установка соееиннения с источником } \\
\text { Установка соееиинения с назначением } \\
\text { Проверка } \\
\text { Подготовка к выполненнию } \\
\text { До выполнения } \\
\text { Выполнение } \\
\text { (i) Копирование в [dbo].[Пист1s] } \\
\text { После выполнения }\end{array}$}} & Успешно & & & & \\
\hline & & $\begin{array}{l}\text { Успешно } \\
\text { Успешно }^{-1}\end{array}$ & & & & \\
\hline & & Успешно & & & & \\
\hline & & $\begin{array}{l}\text { Успешно } \\
\text { Успешно }\end{array}$ & & & & \\
\hline & & & Передано стоок: $53 . .$. & & & \\
\hline \multicolumn{4}{|r|}{ Отет -1} & & & \\
\hline \multicolumn{4}{|r|}{ 3акрыть } & & & \\
\hline & Name & \multicolumn{2}{|c|}{ Name of Product } & & Version of Product & Name of Company \\
\hline 400 & IVMIT-14-910-... & \multicolumn{3}{|c|}{ Microsoft SQL Server Data Tools Build Utilities - RUS... } & 11.1.20627.00 & Microsoft Corporation \\
\hline 401 & IVMIT-14-910-... & \multicolumn{3}{|c|}{ Microsoft SQL Server Database Publishing Wizard 1.3} & 10.0.1600.22 & Microsoft Corporation \\
\hline 402 & IVMIT-14-910-... & \multicolumn{3}{|c|}{ Microsoft SQL Server Native Client } & 09.00 .3042 .00 & Microsoft Corporation \\
\hline 403 & IVMIT-14-910-... & \multicolumn{3}{|c|}{ Microsoft Sync Framework Runtime v1.0 (x64) ru } & 01.0 .1215 .0 & Microsoft Corporation \\
\hline 404 & IVMIT-14-910-... & \multicolumn{3}{|c|}{ Microsoft Sync Services for ADO.NET v2.0 (x64) ru } & 02.0.1215.0 & Microsoft Corporation \\
\hline 405 & IVMIT-14-910-... & \multicolumn{3}{|c|}{ Microsoft System CLR Types для SQL Server 2012} & 11.0.2100.60 & Microsoft Corporation \\
\hline 406 & IVMIT-14-910-... & \multicolumn{3}{|c|}{ Microsoft System CLR Types для SQL Server 2012 (... } & 11.0.2100.60 & Microsoft Corporation \\
\hline 407 & IVMIT-14-910-... & \multicolumn{3}{|c|}{ Microsoft Visual C++ 2005 Redistributable } & 08.0.61001 & Microsoft Corporation \\
\hline 408 & IVMIT-14-910-... & \multicolumn{3}{|c|}{ Microsoft Visual C++ 2005 Redistributable (x64) } & 08.0 .61000 & Microsoft Corporation \\
\hline 409 & IVMIT-14-910-... & \multicolumn{3}{|c|}{ Microsoft Visual C ++2008 Redistributable - x64 9.0.3... } & 09.0 .30729 .4148 & Microsoft Corporation \\
\hline 410 & IVMIT-14-910-... & \multicolumn{3}{|c|}{ Microsoft Visual C++ 2008 Redistributable - x64 9.0.3... } & 09.0 .30729 .6161 & Microsoft Corporation \\
\hline 411 & IVMIT-14-910-... & \multicolumn{3}{|c|}{ Microsoft Visual C++ 2008 Redistributable - x86 9.0.3... } & 09.0 .30729 .4148 & Microsoft Corporation \\
\hline 412 & IVMIT-14-910-... & \multicolumn{3}{|c|}{ Microsoft Visual C ++2008 Redistributable - $x 86$ 9.0.3... } & 09.0.30729.6161 & Microsoft Corporation \\
\hline 413 & IVMIT-14-910-... & \multicolumn{3}{|c|}{ Microsoft Visual C ++2010 x64 Redistributable - 10.0... } & 10.0.40219 & Microsoft Corporation \\
\hline 414 & IVMIT-14-910-... & \multicolumn{3}{|c|}{ Microsoft Visual C $++2010 \times 86$ Redistributable $-10.0 \ldots$} & 10.0.40219 & Microsoft Corporation \\
\hline 415 & IVMIT-14-910-... & Microsoft Vis & al C++ $2017 \mathrm{Re}$ & ble $(x 64)-14 \ldots$ & 14.11.25325.0 & Microsoft Corporation \\
\hline 416 & IVMIT-14-910-... & Microsoft Vis & ala $C_{++} 2017 R_{e}$ & ble $(\times 86)-14 \ldots$ & 14.11.25325.0 & Microsoft Corporation \\
\hline
\end{tabular}

Fig.7. Fragment of the result of a table join query

In the dbo.KS table we have 823006 software products.

At the final stage, a request was created, which among all the identical software products selects the product with the largest version. A new table with unique lines was formed, in which all software products are taken with the maximum version. (Fig. 8)

Select [Diploma (Finished Plates)]. Dbo.KS. [Name], [Diploma (Finished Plates)]. Dbo.KS. [Product Name], [Diploma (Finished Plates)].
Dbo.KS. [Company Name], MAX ([Diploma (Finished Plates)]. Dbo.KS. [Product Version]) as INTO Version [Diploma (Finished Plates)]]. Dbo. [Finished Table] FROM [Diploma (Ready plates)]. Dbo.KS GROUP BY [Diploma (Finished plates)]. Dbo.KS. [Name], [Diploma (Finished plates)]. Dbo.KS. [Product name], [Diploma (Finished plates)]. Dbo.KS. [Company name] HAVING COUNT( $(*)>=1$

ORDER BY [Diploma (Ready plates)]. Dbo.KS. [Name] 
REVISTA SAN GREGORIO, 2019, No.32, SPECIAL ISSUE AUGUST, ISSN: 1390-7247; EISSN: 2528-7907

\begin{tabular}{|c|c|c|c|c|}
\hline & Name & Name of Product & Name of Company & Version \\
\hline 562 & 10TD-1... & Microsoft Lync 2010 & Корпорация Майкрософт & 04.0 .7577 .0 \\
\hline 563 & 1OTD-1... & Microsoft Outlook & Microsoft Corporation & $14.0 .7172 .5 \ldots$ \\
\hline 564 & 1OTD-1... & Microsoft Setup Bootstrapper & Microsoft Corporation & $14.0 .7011 .1 \ldots$ \\
\hline 565 & 10TD-1... & Microsoft Visual C++ 2005 Redistributable (x64) & Microsoft Corporation & 08.0 .61000 \\
\hline 566 & 1OTD-1... & Microsoft Visual C++ 2008 Redistributable & Microsoft Corporation & $09.0 .30729 \ldots$ \\
\hline 567 & 10TD-1... & Microsoft@ Office & Microsoft Corporation & 14.0 .0370 .400 \\
\hline 568 & 10TD-1... & $\mathrm{MPC} \cdot \mathrm{HC}$ & MPC-HC Team & $01.7 .10 .290 \ldots$ \\
\hline 569 & 10TD-1... & WinScan2PDF SoftwareOK.com & Nenad Hrg (SoftwareOK.com) & $2,7,1,0$ \\
\hline 570 & 10TD-1... & 7-Zip $16.04(x 64)$ & Igor Pavlov & 16.anp \\
\hline 571 & 10TD-1... & Adobe Acrobat Update Service & Adobe Systems Incorporated & $1,7,0,0$ \\
\hline 572 & 10TD-1... & Adobe Flash Player 24 NPAPI & Adobe Systems Incorporated & $24 \cdot 0.0 .194$ \\
\hline 573 & 10TD-1... & Adobe Installation Helper & Adobe & 02.0 .2 .13 \\
\hline 574 & 10TD-1... & BGInfo & Sysintemals & 4. $16,0,0$ \\
\hline 575 & 10TD-1... & Bootstrapper Small & Adobe Systems Incorporated & 11.0.0.379 \\
\hline 576 & 1OTD-1... & FineReader & ABBYY. & 11.0 .102 .481 \\
\hline 577 & 1OTD-1... & FineReader Tech & ABBYY. & 11.0.1002.329 \\
\hline 578 & 10TD-1... & Google Update & Google LLC & 01.3 .34 .7 \\
\hline 579 & 10TD-1... & Kaspersky Security Center & AO Kaspersky Lab & 10.4 .6000 .0 \\
\hline 580 & 10TD-1... & K-Lite Mega Codec Pack 12.8.5 & $\mathrm{KLCP}$ & 12.08 .2005 \\
\hline 581 & 10TD-1... & Kmlnstall Core Module & KYOCERA Document Solutions I... & 05.1 .1202 \\
\hline 582 & 10TD-1... & Kyocera Product Library & KYOCERA Document Solutions I... & 05.0.1120 \\
\hline 583 & 10TD-1... & Kyocera TWAIN Driver & KYOCERA Document Solutions I... & 02.0 .3404 \\
\hline 584 & 10TD-1... & Microsoft .NET Framework 4.6.1 & Microsoft Corporation & 04.6 .01055 \\
\hline 585 & 10TD-1... & Microsoft Equation Editor & Design Science, Inc. & 03. \\
\hline 586 & 10TD-1... & Microsoft Visual C++ 2008 Redistributable -x86 9.0.3... & Microsoft Corporation & $09.0 .30729 \ldots$ \\
\hline 507 & $1 \cap \operatorname{Tn} 1$ & 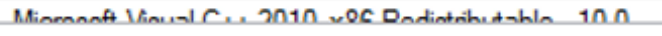 & Minmonf Camantion & 100.10210 \\
\hline
\end{tabular}

Fig. 8. Query result fragment

\section{Summary}

Thus, a comparative integration of the two software tools for auditing the Microsoft System Center Configuration Manager and Kaspersky Security Center software was performed. As a result of all the above actions, a database was obtained in which all information about the software of the Kazan (Volga Region) Federal University, collected from two sources of information collection, was collected.

\section{Conclusion}

It can be said that Microsoft System Center Configuration Manager accumulates more information than Kaspersky Security Center. The request to SCCM produced 533178 lines, and the $\mathrm{KSC}$ request was 208557 lines less. However, KSC collects information more accurately than SCCM. Using these two products allows you to collect information about the installed software of the organization most accurately and extensively.

\section{Acknowledgments}

The work is performed according to the Russian Government Program of Competitive Growth of Kazan Federal University.

\section{References}

[1]. Information technology audit: A textbook for universities / Grekul V.I. - M.: Gore. Line-Telecom, 2015. - 154 p.

[2]. Managing a Microsoft Lifecycle Policy with the Configuration Manager [Electronic Resource]. - Access Mode:

https://docs.microsoft.com/ru-

$\mathrm{ru} / \mathrm{sccm} / \mathrm{core} / \mathrm{clients} / \mathrm{manage} /$ asset-

intelligence/product-lifecycle-dashboard (Contact Date: 05/06/2019)

[3]. Kaspersky Security Center [Electronic resource]. - Access Mode: https://www.kaspersky.com/small-tomedium-business-security/security-center (Contact Date: 05/06/2019)

[4]. Kaspersky Security Center [Electronic resource]. - Access mode: http://www.csoft.ru/catalog/soft/kasperskysecurity-center/kaspersky-securitycenter.html

(Date of appeal 05/05/2019)

[5]. Textbook for students of institutions of secondary vocational education / G. S. Gokhberg, A. V. Zafievsky, A. A. Korotky. 8th ed. M: Publishing center "Academy", 2013. - 208 p.

[6]. Programming Languages: Tutorial / OL. Golitsyna, T.L. Partyka, I.I. Popov. - 3rd ed., 
Pererab. and add. - $\mathrm{M}$.: Forum: SIC INFRAM, 2017. - 400 p.

[7]. Information technology: Textbook / O.L. Golitsyna, N.V. Maksimov, T.L. Partyka, I.I. Popov. - 2nd ed., Pererab. and add. - M .: Forum: INFRA-M, 2008. - 608 p.

[8]. Technical means of informatization: Textbook / N.V. Maksimov, T.L. Partyka, I.I. Popov. - 4th ed., Pererab. and add. - M .: Forum: SIC INFRA-M, 2013. - 608 p.

[9]. Audit: theory and practice: Textbook: In 2 parts Part 1: Theory / L.I. Voronin. - 4th ed., Pererab. and add. - M.: INFRA-M, 2018. - 314 p.

[10]. Audit: Studies. manual / S.M. Bychkova, E.Yu. Itygilova; Ed. IM IN. Sokolova. - M .: Master, 2009. - 463 p.

[11]. Audit: Uch. / Fedorenko I.V., Zolotareva G.I. - M .: NIC INFRA-M, 2016. $-272 \mathrm{p}$.

[12]. Audit: Workshop: Study Guide / N.V. Parushin, S.P. Suvorov, E.V. Galkina. - 3rd ed., Pererab. and add. - M .: ID FORUM: SIC INFRA-M, 2013. - 288 p.

[13]. SQL - the language of relational databases: Tutorial / Kara-Ushanov V.Yu., 2nd ed., Sr. - M.: Flint, Publishing House Ural. Un-one, 2017. - 156 p.

[14]: Kultigin, O. P. Database administration. DBMS MS SQL Server [Electronic resource]: studies. allowance / O. P. Kultigin. - M : MFPA, 2012. - 232 p.

[15]. Microsoft SQL Server 2012: Manual / Bondar AG - St. Petersburg: BHVPetersburg, 2013. - 608 p.

[16] Baykalova, E. D., Artyna, M. K., Dorzhu, N. S., Ochur, T. K., \& Mongush, D. S. (2018). Morphological interference in the process of mastering English speech in conditions of interaction of Tuvan, Russian and English as a foreign language. Opción, 34(85-2), 35-60.

[17] Parvizian, F., Ghojavand, K., \& Niknejadi, F. (2015). Effectiveness of Emotional Intelligence on Emotional Alexithymia of Married Women Teachers in Yasuj City. UCT Journal of Social Sciences and Humanities Research, 3(1), 32-35. 